\title{
COMPORTAMENTO DOS JOVENS CONSUMIDORES DE TELEFONIA CELULAR: ANÁLISE DAS METAS DE CONSUMO ${ }^{1}$
}

\section{BEHAVIOR OF THE YOUNG CONSUMERS OF CELLULAR TELEPHONY: ANALYSIS OF THE CONSUMPTION GOALS}

\author{
Danielle Mantovani Lucena da Silva ${ }^{2}$ \\ Paulo Henrique Muller Prado ${ }^{3}$
}

\begin{abstract}
Resumo: Tendo em vista a necessidade de investigar os pressupostos que levam o consumidor a optar por certos produtos e serviços, o presente estudo tomou como base as metas de consumo no nível de "ter", proposta por Ratneshwar et al (2000) para identificar uma taxonomia de jovens consumidores de telefone celular a partir das suas preferências por atributos e dos benefícios buscados nesses atributos. A pesquisa teve caráter eminentemente exploratório e se baseou em uma amostragem por conveniência e julgamento. Em uma primeira fase foram feitas entrevistas qualitativas e identificados os principais atributos e benefícios associados à avaliação de alternativas de telefonia celular. $\mathrm{Na}$ seqüência, a realização de um survey com 317 pesquisados e as posteriores Análises Fatoriais revelaram cinco grupos de atributos - funções básicas, funções avançadas, tipo de plano, operadora e design. A partir das preferências por atributos foi realizada uma Análise de Cluster e foram encontrados três grupos distintos. Por fim, a ANOVA e o Teste Qui Quadrado foram utilizados na discriminação dos grupos encontrados. A taxonomia apresentada sugere a presença de três clusters, denominados Tecnológicos, Focados no Serviço e Utilitários.
\end{abstract}

Palavras-chave: Metas de Consumo; Jovens; Telefonia Celular.

Abstract: The need to investigate the conditions that lead consumers to choose certain products and services, this study has taken as the basic goals of consumption in the level of "have", the study proposed by Ratneshwar et al (2000) to identify a taxonomy of young consumers of mobile phone from their preferences on attributes and benefits sought in such attributes. The research was exploratory and based on a survey of convenience. In the first phase were carried out qualitative interviews and identified the key attributes and benefits associated with the evaluation of alternatives cellular telephone. After that, were collected 317 questionnaires and the subsequent analysis showed satisfactory five groups of attributes - basic functions, advanced functions, type of plan, operating and design. From the attributes' preferences an analysis of Cluster was carried out and found three distinct groups. Finally, the ANOVA and chi square test were used for discrimination of the groups found. The groups were named "Technology focused", "Service focused" and "Utilitarians".

Key-words: Consumption goals; Youngers; Cellular Phones.

\footnotetext{
${ }^{1}$ Artigo Recebido em 21.09.2008 . Revisado por pares em 05.11.2008. Recomendado em 02.02.2009 por Denise Del Pra Netto Machado (editora). Publicado em 02.03.2009.

Organização Responsável pelo periódico: Universidade regional de Blumenau - FURB - www.furb.br/rn
}

\footnotetext{
${ }^{2}$ Doutoranda em Administração pela UFPR-dm lucena@yahoo.com.br

${ }^{3}$ Professor da Universidade Federal do Paraná - pprado@ufpr.br
} 


\section{INTRODUÇÃO}

Os fatores que influenciam o processo de escolha de produtos e serviços têm sido de grande interesse para os pesquisadores. Identificar suas preferências, desvendar suas atitudes e os fatores que os levam a adquirir determinadas marcas de certas categorias de produtos tem levado a um grande número de investigações (CHERNEV, 2005; COUPEY, 1994; DHAR et al 1999; THOMPSON et al, 2005).

Os consumidores tendem a selecionar a opção que melhor se aplica às suas metas de consumo, maximizando assim, a eficiência da escolha (BETTMAN; LUCE; PAYNE, 1998). Isto significa que eles farão a melhor escolha possível, tendo em vista seus objetivos e limitações de consumo. Como apontam Desai e Hoyer (2000), as metas de consumo são fatores de direcionamento para a aquisição de informação, processamento da mesma e escolha do consumidor. Desse modo, os critérios utilizados para avaliar os atributos levarão em conta os benefícios esperados.

Além disso, Gutman (1997) argumenta que durante o processo de avaliação de alternativas, o consumidor normalmente tende a buscar informação sobre os benefícios de um produto específico, e sua motivação principal não é determinada pelos atributos físicos deste produto, mas pelos benefícios que esses atributos oferecem.

Considerando as metas de consumo do nível "ter", que de acordo com Ratneshwar et al (2000) focam no desejo de um indivíduo de possuir um determinado produto ou marca por causa dos atributos e características associadas ao mesmo, pretende-se identificar quais são os atributos e benefícios buscados pelos jovens consumidores de telefonia celular. Além disso, o estudo propõe uma taxonomia de jovens consumidores de telefone celular a partir das suas preferências por atributos e dos benefícios buscados nesses atributos.

Nesta pesquisa é analisada a telefonia celular móvel, considerando tanto o produto (aparelho), quanto o serviço prestado pela operadora. Tal decisão foi tomada com base na percepção de que o consumidor realiza desses durante a avaliação de alternativas, observada posteriormente na etapa exploratória da pesquisa.

Embora se tenha conhecimento de que muito do comportamento de compra seja orientado por metas, sendo as escolhas feitas como forma de atingir determinados objetivos, não se sabe até que ponto essas metas são realmente satisfeitas, principalmente ao serem considerados produtos de alta tecnologia, onde o conhecimento sobre a utilização e o desempenho dos atributos pode variar de pessoa para pessoa. Dessa forma, a proposição de uma taxonomia que ajude a compreender quais os atributos e benefícios buscados pelo consumidor pode ser de grande relevância para a formulação de estratégias que facilitem a avaliação de alternativas pelo consumidor. Tal proposta visa contribuir para o debate sobre como os consumidores buscam atingir as suas metas de consumo e como essas diferenças podem ser úteis para a análise da segmentação de mercado tanto sob a ótica acadêmica quanto gerencial.

De acordo com a HSM Management (2006), sobretudo para os consumidores jovens, que costumam ser entusiastas de novas tecnologias, o telefone celular tornou-se bastante comum no cotidiano, fazendo uso dos aparelhos móveis para fazer contatos sociais, jogar e fazer download de músicas e ringtones (toques para celular). Ainda de acordo com a reportagem, nesse segmento os telefones celulares se tornaram símbolos de status, capas personalizadas, entre outros itens, assemelhando-se nesse sentido, a roupas, acessórios e outros elementos de expressão e de moda (HSM MANAGEMENT, 2006).

Os jovens brasileiros gastam mais de 10 bilhões de reais somente com telefonia celular por ano (TELECO, 2006). Dados apresentados pela Veja (2005), afirmam que os jovens se empenham para estar atualizados com as novas tecnologias, $71 \%$ têm telefone celular, $41 \%$ já trocaram de aparelho celular de duas a três vezes, $46 \%$ possuem computador e $53 \%$ afirmam que seu objeto de desejo é uma câmera digital. 
De acordo com a Associação Brasileira de Telecomunicações (Telebrasil), a indústria do celular procura aumentar a receita por usuário com serviços de mensagem, multimídia, gráficos, tons de chamada, jogos e, obviamente, televisão e vídeo móvel. Por outro lado, as operadoras celulares, hoje, se defrontam com um cenário de investimentos a amortecer, grandes ativos a serem utilizados e alta competição, necessitando focar em serviços básicos, geradores de receita - voz e mensagens curtas que geram entre 90 a $95 \%$ da receita - e oferecer ao mercado pacotes de serviços a preços fixos e baratos.

Os critérios utilizados para selecionar as alternativas de compra, por exemplo, podem fornecer subsídios para o desenvolvimento de estratégias de comunicação específicas para esse público.

\section{METAS DE CONSUMO}

De acordo com Bagozzi e Dholakia (1999), os consumidores efetuam suas compras para produzir um estado ou meta final. Uma grande parte do comportamento do consumidor é voltada para metas de consumo, sendo essas metas focadas em um resultado específico que pode ser alcançado através do consumo. Os autores argumentam que as metas não estão limitadas a estados finais, podendo abranger experiências, seqüências de acontecimentos interconectados e processos em andamento.

As metas de consumo provêem motivação primária e são fatores de direcionamento para a aquisição de informação, processamento da mesma e escolha do consumidor. Além disso, os consumidores categorizam mentalmente produtos através da sua capacidade de satisfazer metas salientes (RATNESHWAR; MICK, 2005; DESAI; HOYER, 2000; DESAI; RATNESHWAR, 2003; ).

Ratneshwar et al (2000) propõem uma categorização de três níveis distintos de motivação, desenvolvendo uma estrutura de metas dinâmica:

- Metas no nível "ter": focam no desejo de um indivíduo de possuir um determinado produto ou marca por causa dos atributos e características associadas ao mesmo. Estas metas refletem o desejo do consumidor de possuir produtos com determinadas características e benefícios.

- Metas do nível "fazer": focam no desejo de um indivíduo de buscar um resultado desejado em uma determinada situação. Muitas metas, muitas vezes, são apenas parte da rotina do ser humano e refletem as preocupações e intenções de consumo.

- Metas do nível "ser": engloba os mais abstratos objetivos de uma estrutura de metas de um indivíduo. Neste nível, as metas pertencem aos valores e temas de alguém.

Os níveis "ter" e "ser" tendem a ser moldados pelos contextos no qual o consumidor procura preencher as metas do nível "fazer". Huffman e Houston (1993) realizaram um estudo buscando os antecedentes das metas como a informação prévia sobre o produto e analisaram também a escolha e o feedback de informações adquiridas após a escolha do produto. Os resultados apontam que as informações que os consumidores aprendem são organizadas na memória em torno de metas que direcionam as experiências de consumo.

No presente estudo serão consideras apenas as metas no nível "ter", que englobam os benefícios buscados e a preferência por atributos, pois pretende-se fazer a relação entre essas duas variáveis e os critérios de avaliação utilizados pelos consumidores no processo de escolha, verificando se as metas foram alcançadas nesse sentido. De acordo com Ratneshwar et al (2000), a busca por benefícios é entendida pelas conseqüências desejadas através da compra de um produto e a preferência por atributos como características físicas do produto que possuem preferência pelo consumidor. 


\subsection{Preferência Por Atributos e Benefícios Buscados}

A preferência por certos atributos advêm das metas do consumidor, e, por isso, normalmente são estabelecidos alguns critérios de avaliação. De acordo com Solomon (2002, p.220), "critérios de avaliação são as dimensões a serem utilizadas para julgar os méritos de opções concorrentes". Os critérios que os consumidores usam para avaliar as alternativas que formam seu conjunto de consideração são expressos em termos de importância dos atributos do produto.

Quanto à influência dos atributos na intenção de compra do consumidor é possível destacar que são considerados três tipos de atributos: (a) atributos característicos, que descrevem as propriedades físicas dos produtos; (b) os atributos benéficos, que se referem à utilidade a ser proporcionada quando o produto é consumido, e; (c) atributos de imagem, que indicam ostentação por parte do consumidor e simbolizam a imagem que o consumidor deseja transmitir. (LEFKOFF-HAGIIJS; MANSON, 1993).

Existem diversos fatores que podem influenciar a preferência por certos atributos como os traços típicos de uma pessoa e sua personalidade, além de influências sociais, culturais, psicológicas e dos meios de comunicação. Mackenzie (1986), por exemplo, verificou que a propaganda pode influenciar a avaliação do consumidor quanto à relevância dos atributos, pois ela pode direcionar a atenção para certos aspectos do produto, recebendo maior importância pelo consumidor ao serem enfatizados. Dessa forma, pode-se concordar com Engel et al (2000) ao observarem que os atributos de um produto tendem a variar substancialmente na importância que eles têm para os consumidores quando estes formam suas atitudes sobre os produtos.

Alpert (1971) preocupou-se em conhecer quais são os atributos que efetivamente determinam a aquisição de bens e serviços. Baseando-se em alguns estudos realizados para medir o grau de influência dos atributos na intenção de compra de bens e serviços, o autor verificou que a simples identificação de atributos considerados importantes não permitia conhecer dentre eles quais determinavam a compra e a preferência por um determinado produto ou marca.

Alguns atributos, dentro do grupo classificados como importantes, pareciam possuir um potencial maior de influencia no momento da escolha. Baseando-se no fato de que nem todos os atributos são igualmente importantes na definição das preferências do consumidor, Alpert (1971) faz uma distinção entre atributos determinantes e não determinantes. Além disso, o autor apresenta um método para testar abordagens que identifiquem os atributos mais relevantes e que exercem maior influência na escolha e preferência do consumidor.

Independente da relevância do atributo, Zeithaml (1988) classifica os atributos como: (a) atributos intrínsecos, associados a características físicas e funcionais do produto, como resistência, tamanho, cor e design, não podendo ser alterados sem mudar a natureza do produto, e; (b) atributos extrínsecos, não são associados a características físicas do produto, como o preço, a propaganda e a marca, e, portanto, qualquer alteração nestes atributos não implicaria em modificação no produto físico.

A autora aponta ainda que ambos os tipos de atributos são proveitosos para a avaliação da marca e da qualidade, porém os atributos intrínsecos são utilizados quando o consumidor já tem em mente conhecimento sobre o produto, ao contrário dos atributos extrínsecos, que são utilizados quando o consumidor possui pouco conhecimento ou informação relativa ao produto (ZEITHAML, 1988).

Os benefícios, segundo Weinstein (1995), constituem o conjunto das vantagens ou satisfações que um produto proporciona às necessidades ou desejos dos consumidores, indo além das características do produto. Servem, assim, para satisfazer as necessidades físicas, emocionais ou psicológicas do consumidor. 
De forma mais específica, Vriens e Ter Hofstede (2000) definem os benefícios como sendo características pouco, ou não, diretamente observáveis de um produto ou marca, podendo ser a combinação de diversos atributos. Os benefícios são o resultado da utilização do produto pelo consumidor, como facilidade de uso, conforto e conveniência, por exemplo.

Mowen e Minor (2003) diferem os atributos dos benefícios afirmando que enquanto os atributos podem ser definidos como aspectos ou características do produto, os benefícios são os resultados positivos que o produto proporciona ao consumidor. Wu (2001) complementa ao afirmar que benefícios são uma soma de vantagens ou satisfações trazidas por um produto que conseguem suprir as necessidades ou desejos de um indivíduo.

De acordo com Gutman (1997), ao avaliar um produto ou serviço, o consumidor normalmente tende a buscar informação sobre os benefícios de um produto específico, e sua motivação principal não é determinada pelos atributos físicos deste produto, mas pelos benefícios que os atributos oferecem. $\mathrm{O}$ autor afirma que essa forma de raciocínio utilizada pelo consumidor resulta de um processo baseado no modelo teórico de expectativas e valores do consumidor.

Dessa forma, as ações do consumidor para a escolha geram resultados que farão com que ele aprenda quais ele deve evitar ou desejar e que guiarão suas escolhas futuras. Por meio desse processo de aprendizagem, o consumidor passa a pensar em um produto ou serviço de acordo com seus atributos, as conseqüências relacionadas ao seu uso e a sua instrumentalidade para o alcance de valores pessoais relevantes. (GUTMAN, 1997).

Lin e Fu (2001) fazem a conexão entre metas e conseqüências do produto, apresentando uma divisão baseada em conseqüências funcionais do produto e psicológicas, onde a primeira refere-se a atributos tangíveis como cor e tamanho, enquanto que a segunda está ligada a características mais abstratas como estilo e qualidade, que podem envolver um grupo de atributos para uma só característica.

\section{METODOLOGIA}

Com o objetivo de identificar os atributos e benefícios relacionados ao que tange à telefonia celular móvel, o público-alvo escolhido foram os jovens, que de acordo com Ferreira (2003), representam um segmento de mercado com grande potencial de consumo. Como abordados em estudos anteriores (FERREIRA, 2003; PÁDUA JÚNIOR e PRADO, 2005), o público entre 17 e 25 anos representa um segmento de mercado em expansão, potenciais consumidores de telefonia celular. Assim, o método utilizado para o desenvolvimento da pesquisa segue as recomendações de Malhotra (2001) e Churchill (1999), no que tange às fases do levantamento de corte transversal, assim como aquelas sugeridas por Hair et al (2005), quanto ao uso de técnicas de análise multivariadas para avaliação da relação entre as variáveis.

O estudo foi desmembrado em duas fases distintas onde foram utilizados os métodos qualitativos e quantitativos de pesquisa, baseado em conceitos como os de Churchill (1999), Aaker et al (2004) e Malhotra (2001). O universo da pesquisa foi definido como: jovens universitários, com idade entre 17 e 25 anos, residentes em Curitiba, pertencentes às classes sócio-econômicas A, B e C, de acordo com o Critério de Classificação Sócio-econômica Brasil. A amostra foi de respondentes que tivessem telefone celular e que participaram da escolha do produto.

Foram realizadas entrevistas em profundidade com 12 consumidores que compuseram o perfil da amostra e também com dois especialistas da área de telefonia. As entrevistas com profissionais da área de telefonia celular tiveram o intuito de obter informações sobre o mercado e os serviços oferecidos especialmente para o público jovem, tendências do mercado e novos serviços que estavam entrando em operação. A partir da transcrição das entrevistas, sua análise foi feita por meio de uma categorização das variáveis. Após a elaboração do 
instrumento de pesquisa, foi realizado um pré-teste para uma amostra dividida em dois grupos de 12 pessoas, com a finalidade de verificar a necessidade de eventuais correções no questionário.

A coleta quantitativa foi realizada com turmas de graduação de instituições de ensino superior em Curitiba. O tamanho da amostra levou em consideração a natureza da pesquisa, as variáveis abordadas e as técnicas estatísticas utilizadas para a posterior análise dos dados. No total, foram obtidos 317 questionários válidos. Adotou-se o procedimento de amostragem não-probabilística por conveniência, que de acordo com Malhotra (2001), utiliza seleção aleatória, confiando no julgamento do pesquisador.

Para a validação dos instrumentos de coleta desta pesquisa, foi utilizado o método da validação de conteúdo ou facial (MALHOTRA, 2001), envolvendo o julgamento de experts sobre o conteúdo de uma escala de medida e a avaliação de sua representatividade na mensuração. Neste caso, a validação foi feita por professores da área de marketing de duas instituições de ensino superior em Curitiba.

Os dados quantitativos obtidos foram tratados com análises estatísticas apropriadas, tendo em vista a utilização do software estatístico SPSS versão 13.0. Para identificar os fatores da variável "importância dos atributos" foi utilizada a Análise Fatorial Exploratória (AFE). Para verificar as diferenças entre consumidores na preferência por atributos, inicialmente foi feita uma classificação dos respondentes com base na importância dada a cada um dos fatores verificados na importância dos atributos. Essa classificação foi feita com o auxílio da análise Two-step Cluster. No intuito de identificar as diferenças significantes entre os grupos foi empregada a análise da variância (ANOVA). O teste qui-quadrado revelou quais ligações entre atributos e benefícios eram significativas também na diferenciação entre os grupos. Os resultados obtidos são apresentados na secção seguinte.

\section{RESULTADOS}

A amostra coletada foi composta por $54,3 \%$ de mulheres, entre 20 e 23 anos $(52,3 \%)$, solteiros $(87,1 \%)$ e de classe sócio-econômica B (48,3\%). Estes entrevistados são usuários de telefonia celular a mais de 4 anos $(62,1 \%)$, possuem o aparelho de telefone celular a mais de um ano $(45,1 \%)$, já tendo trocado de aparelho celular no mínimo 3 vezes $(68,9 \%)$. O fato de terem escolhido sozinhos o próprio aparelho apresentou incidência de 55,2\%, utilizando o aparelho majoritariamente para uso pessoal $(50,2 \%)$. Este perfil remonta a um grupo de usuários bastante qualificado, e com familiaridade relativamente elevada aos serviços de telefonia celular.

\subsection{Metas de Consumo no nível "ter" - relações entre atributos e benefícios}

Para mensurar quantitativamente a importância dada aos atributos identificados na fase qualitativa, formulou-se uma questão na qual o respondente indicava o nível de importância dos atributos, desde nenhuma importância (1) até muito importante (5). Foi realizada uma Análise Fatorial Exploratória (AFE) para se determinar a existência de grupos de atributos. Os resultados obtidos para o teste de esfericidade de Bartlett foi de 1251,572 (significância de $0,00)$ e KMO de 0,753 .

Tabela 01 - Fatores Encontrados Para a Variável Importância dos Atributos

\begin{tabular}{l|c|c|c|c|c}
\hline \multicolumn{1}{c|}{ FUNÇÕES } & \multicolumn{5}{c}{ CARREGAMENTOS } \\
\hline Funções Básicas & $\begin{array}{c}\text { Funções } \\
\text { Básicas }\end{array}$ & $\begin{array}{c}\text { Funções } \\
\text { Avançadas }\end{array}$ & Operadora & $\begin{array}{c}\text { Tipo de } \\
\text { Plano }\end{array}$ & Design \\
\hline Agenda Telefônica & & & & & \\
\hline Calendário & 0,779 & & & & \\
\hline
\end{tabular}




\begin{tabular}{|c|c|c|c|c|c|}
\hline Despertador & 0,790 & & & & \\
\hline Mensagem de Texto (SMS) & 0,666 & & & & \\
\hline Vibracall & 0,685 & & & & \\
\hline \multicolumn{6}{|l|}{ Funções Avançadas } \\
\hline Visor Colorido & & 0,599 & & & \\
\hline Câmera Fotográfica & & 0,791 & & & \\
\hline Acesso à Internet & & 0,782 & & & \\
\hline Mp3 Player & & 0,839 & & & \\
\hline \multicolumn{6}{|l|}{ Operadora } \\
\hline Marca do Aparelho & & & 0,646 & & \\
\hline Marca da Operadora & & & 0,812 & & \\
\hline Tipo de Tecnologia & & & 0,664 & & \\
\hline \multicolumn{6}{|l|}{ Tipo de Plano } \\
\hline Pré-pago & & & & 0,855 & \\
\hline Pós-pago & & & & 0,765 & \\
\hline Preço do Aparelho & & & & 0,419 & \\
\hline Área de Cobertura & & & & 0,652 & \\
\hline \multicolumn{6}{|l|}{ Design } \\
\hline Design & & & & & 0,762 \\
\hline Tamanho do Aparelho & & & & & 0,769 \\
\hline $\begin{array}{l}\text { Percentual da Variância } \\
\text { Explicada Após a Rotação }\end{array}$ & $16,03 \%$ & $14,84 \%$ & $9,95 \%$ & $9,84 \%$ & $9,06 \%$ \\
\hline $\begin{array}{l}\text { Percentual da Variância } \\
\text { Acumulada }\end{array}$ & $16,03 \%$ & $30,88 \%$ & $40,83 \%$ & $50,68 \%$ & $59,74 \%$ \\
\hline Alfa de Cronbach & 0,748 & 0,782 & 0,556 & 0,586 & $\mathbf{0 , 5 0 3}$ \\
\hline
\end{tabular}

Fonte: Coleta de dados

Na tabela 01 estão os valores calculados do Alfa de Cronbach para cada um dos fatores. Os valores encontrados indicam uma consistência interna da escala adequada. Os itens que compõem os fatores 1 e 2 , funções básicas e funções avançadas, respectivamente, já foram verificados no estudo de Pádua Júnior e Prado (2005), sendo que as funções básicas representam as tarefas mais comuns em um celular e são mais simples de serem efetuadas. Já as funções avançadas apresentam uma complexidade de utilização maior que as funções básicas.

Observa-se que os valores do Alfa de Cronbach encontrados indicam boa consistência interna apenas para duas dimensões: funções básicas e funções avançadas. As dimensões "operadora", "tipo de plano" e "design" não apresentaram consistência interna de escala adequada. O baixo valor obtido do alfa pode ser, em parte, explicado pela baixa correlação entre os atributos, o que sugere a possível existência de grupos de consumidores que valorizam diferentes atributos. Dessa forma, serão feitas algumas análises para a possível identificação de tipologia de consumidores com base na importância dada a determinados atributos.

$\mathrm{Na}$ aplicação do questionário utilizado para a obtenção dos dados da fase quantitativa do estudo, os respondentes deveriam assinalar a associação entre os atributos e os benefícios sugeridos. Foram apresentados aos respondentes 17 atributos e 14 benefícios, totalizando 238 ligações possíveis. Entretanto, optou-se por trabalhar apenas com as ligações mais significativas em termos da probabilidade de ocorrência. Para isso o corte das ligações foi feita no $80^{\circ}$ percentil, procurando-se atentar as probabilidades mais significativas, uma vez que não foi encontrada qualquer referência na literatura que trate de tal relação de forma probabilística sugerindo valores de referência. Após a determinação das ligações que seriam analisadas, dentre as 238 ligações, restaram 45 ligações. De modo a facilitar a análise, separou-se a discussão dos atributos pelos fatores encontrados na análise fatorial. 


\subsubsection{Funções básicas}

Como proposto por Vriens e Ter Hofstede (2000), os benefícios relacionados ao grupo de "funções básicas" são resultado da utilização do produto pelo consumidor. Neste caso, o principal benefício é a praticidade, que engloba todos os outros benefícios relacionados a esse, como pode ser observado na figura 1.

Figura 1 - Freqüência de Ligações entre as Funções Básicas e Benefícios (\%)

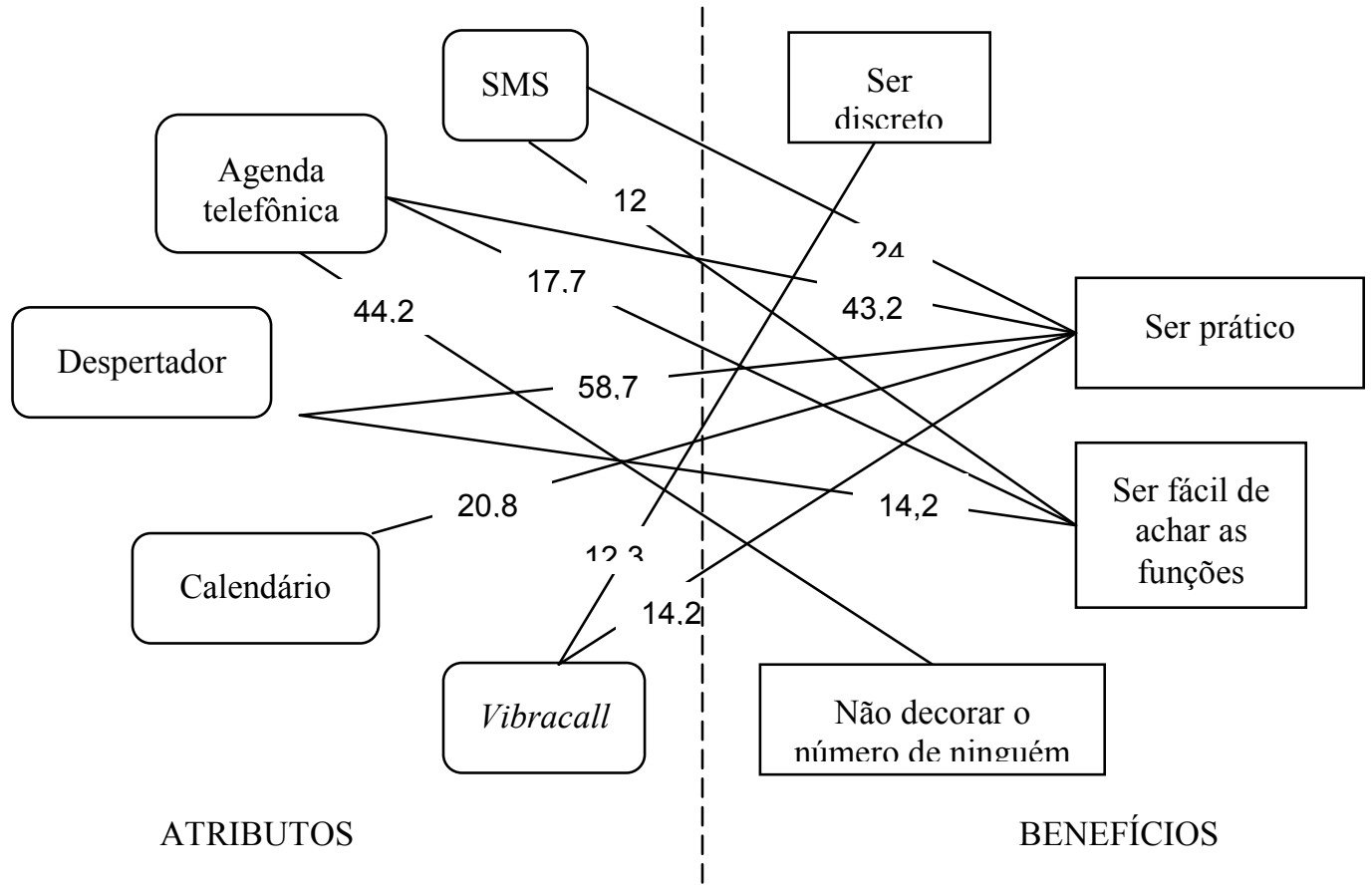

Fonte: Dados da pesquisa

Analisando os benefícios buscados no conjunto de funções básicas, pode-se tomar como base os conceitos de Alpert (1971), para considerar que esses atributos são importantes para praticamente todos os grupos de consumidores, mas ainda não são suficientes para decidir a compra do telefone celular. Entretanto, caso esses atributos estejam ausentes, a avaliação do produto poderá ser comprometida em algum grau.

\subsubsection{Funções avançadas}

A figura 2 a seguir mostra que o grupo composto pelas funções avançadas foi associado não apenas à praticidade, mas também aos benefícios simbólicos, como "ser moderno" e "ser bonito". Neste último caso, esse benefício pode ser associado à forma de utilização do celular pelos jovens, a exemplo de ser mais um "acessório do vestuário". Nesse segmento os telefones celulares se tornaram símbolos de status, com capas personalizadas, entre outros itens, assemelhando-se nesse sentido, às roupas, acessórios e outros elementos de expressão e de moda. 


\section{Figura 2 - Freqüência de Ligações entre as Funções Avançadas e Benefícios (\%)}

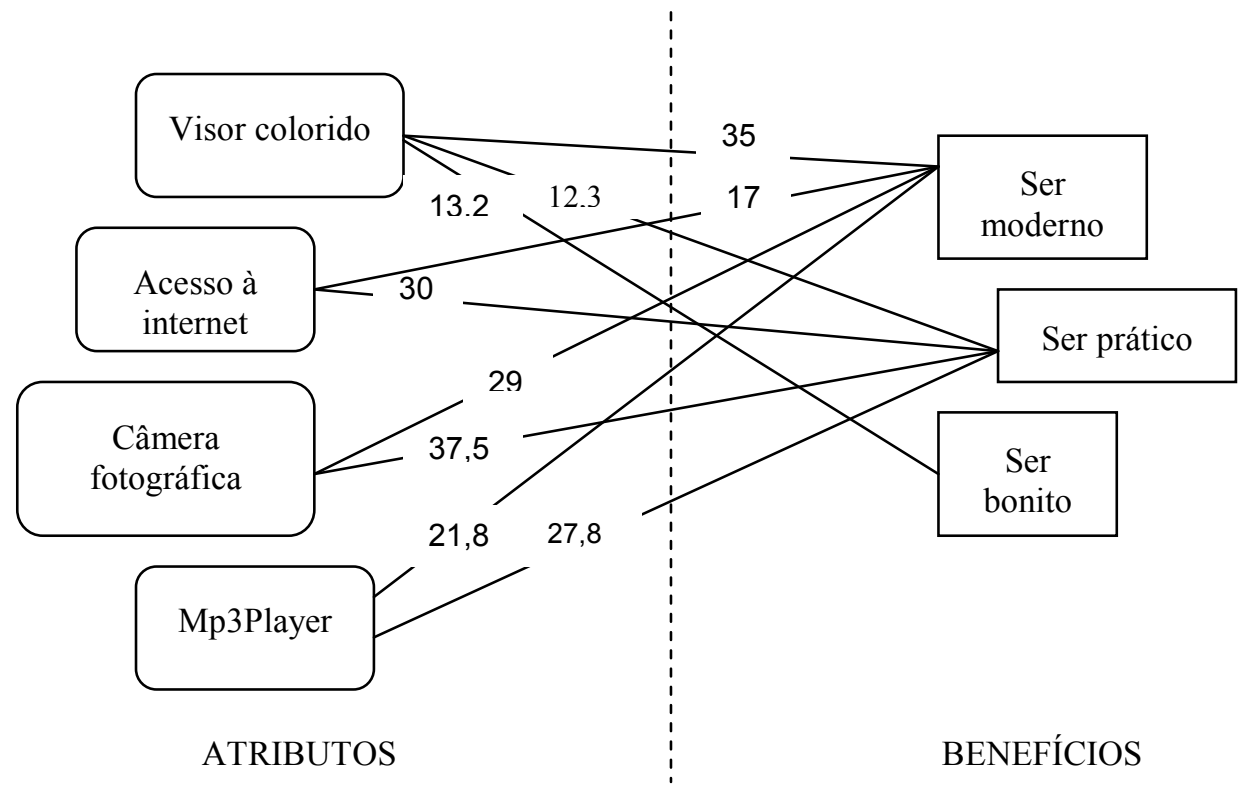

Fonte: Dados da pesquisa

A análise dessas ligações vai de encontro com os argumentos de Gutman (1997), de que a motivação principal do consumidor não é determinada pelos atributos físicos do produto, mas pelos benefícios que eles oferecem. Dessa maneira, as ações dos consumidores para a escolha geram resultados que farão com que ele aprenda quais atributos ele deve evitar ou desejar e que guiarão suas escolhas futuras.

Além disso, esses resultados podem ter alguma relação com os verificados por Mackenzie (1986), onde a propaganda apareceu como um fator influenciador na avaliação do consumidor quanto à relevância dos atributos, podendo direcionar a atenção para certos benefícios do produto, enfatizando-os, sendo então percebidos como importantes. Nesse sentido, as inovações e o aumento do número de funções, principalmente as avançadas tendem a ser associadas a benefícios relacionados à imagem que o consumidor gostaria de passar como ser moderno, por exemplo.

\subsubsection{Operadora}

No que se refere às associações realizadas ao fator "Operadora", a figura 3 aponta que o benefício "ser encontrado" foi associado aos atributos "tipo de tecnologia" (28,1\%), "marca do aparelho" (17\%) e "marca da operadora" (36,9\%). Pode-se inferir que essa relação denota um sentido de preocupação com a qualidade do produto e do serviço prestado pela operadora. De maneira similar ao que pôde ser verificado na fase qualitativa, esses atributos são relevantes na avaliação das alternativas, pois correspondem ao adequado funcionamento do serviço de telefonia móvel.

A escolha da "marca do aparelho" leva em consideração o beneficio "ser fácil de achar as funções que eu procuro", como afirmou 37,5\% dos respondentes. Já a escolha da "marca da operadora" foi associada aos benefícios "estabelecer um limite de gasto mensal" e "possibilidade de receber bônus", de modo que para o atributo "marca da operadora", a ligação com a "possibilidade de receber bônus" foi de 40,7\%. Esses dois benefícios são do 
tipo concreto, e levam em conta basicamente o quanto o consumidor está disposto a gastar para fazer uso do serviço de telefonia.

O único benefício do tipo simbólico verificado nesse grupo foi o "ser moderno", relacionado aos atributos "marca do aparelho" (17,7\%) e "tipo de tecnologia" (10,7\%). A primeira ligação pode ser explicada da mesma forma que a ligação entre "visor colorido" e "ser bonito", pois se o aparelho celular relaciona-se à vaidade por ser visto pelo jovem como um acessório do vestuário, há, desta forma, uma necessidade desse jovem de se sentir bem com o que estão usando.

\section{Figura 3 - Freqüência de Ligações entre o Fator Operadora e Benefícios (\%)}

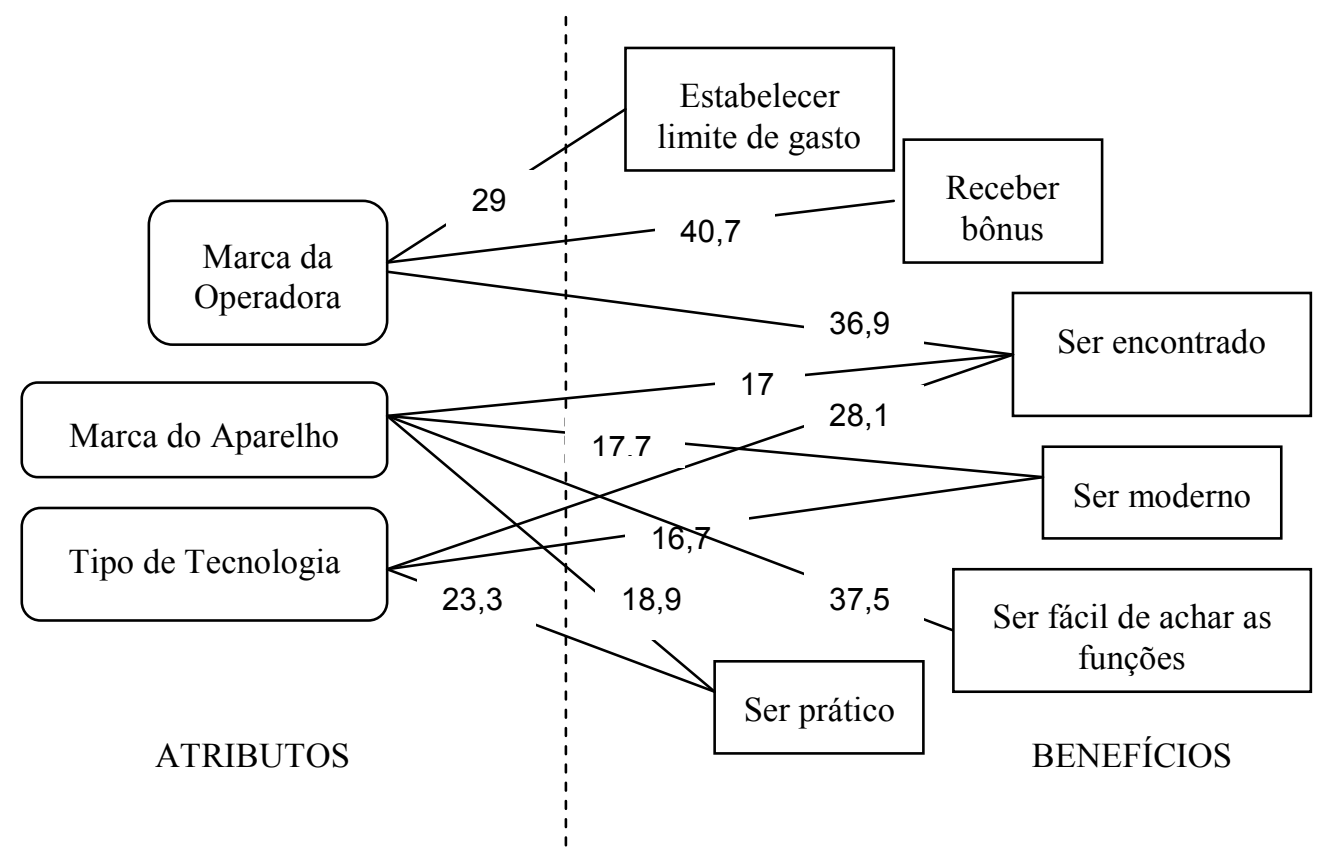

Fonte: Dados da Pesquisa

\subsubsection{Tipo de Plano}

No fator "Tipo de Plano", os dados apresentados na figura 4 apontam forte relação entre o atributo "área de cobertura" e o benefício "permitir que me encontrem" $(55,5 \%)$. O atributo "tipo de plano", aqui consolidando os atributos "ser pré-pago" e "ser pós-pago", apresentou relação representativa com os benefícios "receber bônus" (40,7\%), "estabelecer limite de gasto" $(39,4 \%)$ e "ser prático" $(16,7 \%)$.

Figura 4 - Freqüência de Ligações entre o fator Tipo de Plano e Benefícios (\%)

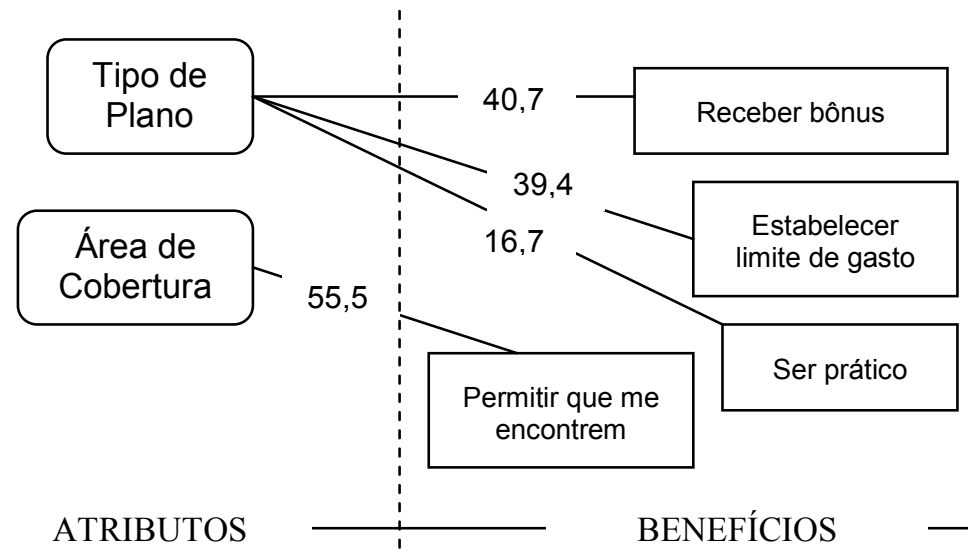

Revista de Negocios, IDSN 19ðU-4431, Blumenau, v. 13, n. 3, p. х1-y/, Julho/Setembro 2008. 90 
Fonte: Dados da Pesquisa

\subsubsection{Design}

Os benefícios relacionados ao atributo "Tamanho do aparelho" foram "ser prático" (25,6\%), "ser discreto" (47,9\%), "não ser motivo de riso porque é grande" $(20,8 \%)$ e "ser fácil de carregar" (34,7\%). Essas relações também estão presentes para o atributo "design", e apresenta benefícios do tipo concreto e simbólico. As ligações entre o "design" e os benefícios concretos foram: "ser prático" $(43,2 \%)$, "ser discreto" $(45,1 \%)$, "ser fácil de carregar" (42\%) e "ser fácil de achar as funções que procuro" $(20,2 \%)$. Esses benefícios correspondem à conveniência e à facilidade de uso do equipamento.

Os benefícios simbólicos que se relacionaram ao "design" foram os seguintes: "não ser motivo de riso" (16,7\%), "ser moderno" (52,7\%), "ser bonito" (18,6\%) e "sentir-se bem" $(22,1 \%)$. Essas relações, mais uma vez, demonstram a preocupação dos jovens com sua aparência e imagem perante os outros.

\section{Figura 5 - Freqüência de Ligações entre o fator Design e Benefícios (\%)}

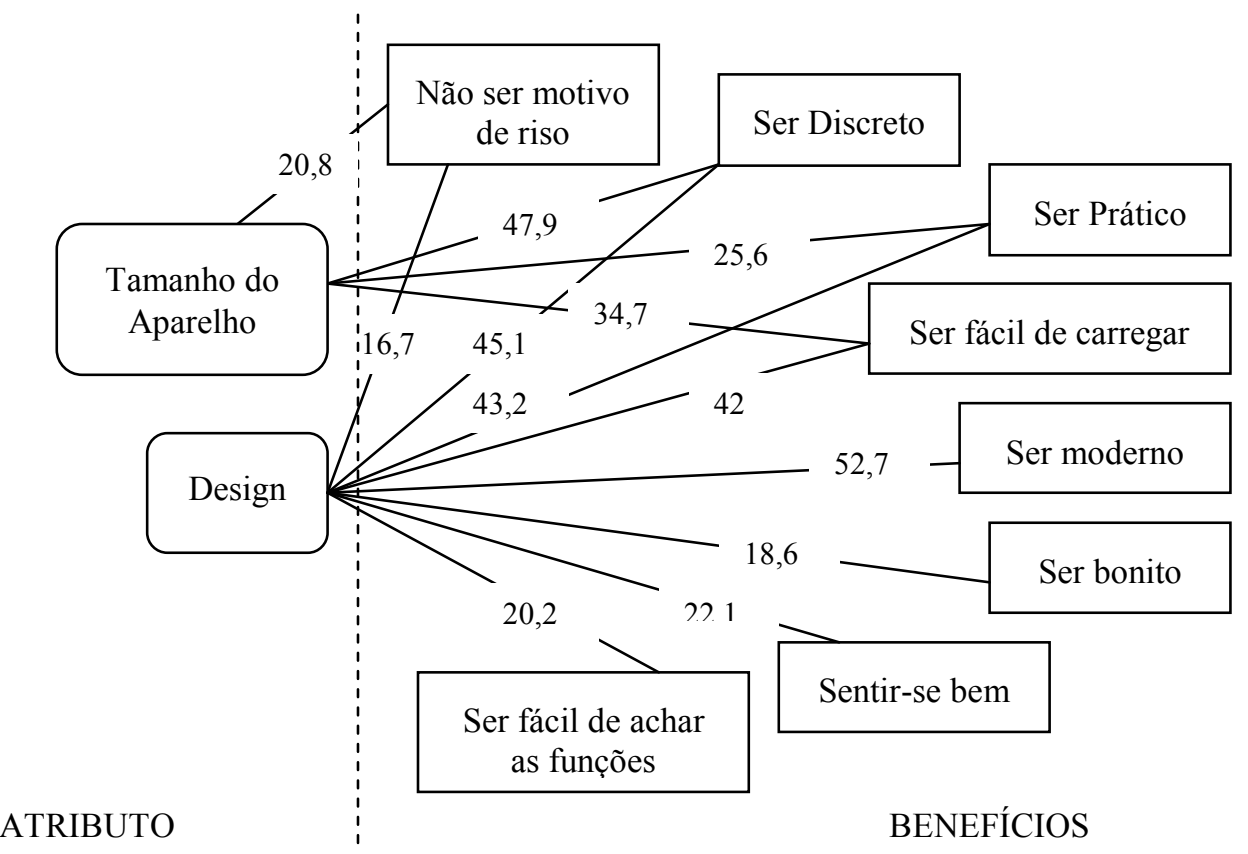

Fonte: Dados da pesquisa

A análise de todos os atributos evidencia que alguns deles apresentaram um número maior de ligações do que outros, não indicando necessariamente que esses são considerados mais relevantes no processo de escolha. Na verdade, cada atributo visa satisfazer um benefício ou um conjunto de benefícios, de modo que a importância relativa dada a cada um deles parte do consumidor.

Nesse sentido, Bettman, Luce e Payne (1998) apontam que o processo de escolha depende de alguns fatores, dentre eles os objetivos do consumidor, de maneira que a opção escolhida proporcione os benefícios que foram buscados nesse processo. Assim, surge a necessidade de propor uma taxonomia de consumidores tendo como base a preferência por atributos e consequentemente, relacionando-os aos benefícios buscados. 


\section{Taxonomia de consumidores com base na Preferência por Atributos}

Para verificar diferenças entre consumidores na preferência por atributos, inicialmente foi feita uma classificação dos respondentes com base na importância dada a cada um dos fatores verificados na importância dos atributos. Essa classificação foi feita com o auxílio da análise Two-step Cluster. O objetivo dessa análise foi o de classificar os respondentes com base na preferência pelos atributos. Foram obtidos 3 clusters: (a) cluster 1, representando $32,5 \%$ da amostra; (b) cluster 2, representando 43,9\%, e; (c) cluster 3, representante de $23,6 \%$ do total de respondentes.

Para verificar as diferenças significantes entre os grupos foi empregada a análise da variância (ANOVA), técnica estatística utilizada para estudar as diferenças entre os valores das médias de duas ou mais populações. A variável dependente é métrica e as variáveis independentes podem ser todas categóricas ou combinações de categóricas e métricas. Verificando a existência de diferenças significativa entre os grupos, foi aplicado o Teste de Tukey para identificar entre quais grupos se encontra esta diferença. (MALHOTRA, 2001).

Baseando-se na comparação das médias entre os grupos encontrados e a preferência por atributos caracterizaram-se os grupos. Tais resultados podem ser vistos na Tabela 02, a seguir.

Tabela 02 - Relações Entre Preferência por Atributos e Clusters

\begin{tabular}{|c|c|c|c|c|c|}
\hline \multirow{2}{*}{$\begin{array}{c}\text { PREFERÊNCIA POR } \\
\text { ATRIBUTOS (FATORES) }\end{array}$} & \multicolumn{3}{|c|}{ CLUSTERS ENCONTRADOS } & \multirow[b]{2}{*}{$\mathrm{F}$} & \multirow[b]{2}{*}{$\mathrm{p}$} \\
\hline & $\begin{array}{c}\text { Cluster } 1 \\
\mathrm{~N}=102\end{array}$ & $\begin{array}{c}\text { Cluster } 2 \\
\mathrm{~N}=138\end{array}$ & $\begin{array}{c}\text { Cluster } 3 \\
\mathrm{~N}=74\end{array}$ & & \\
\hline Funções Básicas & $4,60^{(\mathrm{a})}$ & $4,45^{(b)}$ & $3,70^{(\mathrm{ab})}$ & 65,267 & $0,000^{*}$ \\
\hline Funções Avançadas & $3,56^{(\mathrm{ab})}$ & $2,41^{(\mathrm{a}, \mathrm{c})}$ & $2,92^{(b c)}$ & 44,595 & $0,000^{*}$ \\
\hline Tipo de Plano & $4,49^{(\mathrm{ab})}$ & $4,24^{(\mathrm{ac})}$ & $3,92^{(b c)}$ & 20,064 & $0,000^{*}$ \\
\hline Operadora & $3,52^{(a)}$ & $3,93^{(\mathrm{ab})}$ & $3,63^{(b)}$ & 6,088 & $0,003 *$ \\
\hline Design & $4,58^{(\mathrm{ab})}$ & $3,98^{(\mathrm{a})}$ & $3,83^{(\mathrm{b})}$ & 39,909 & $0,000^{*}$ \\
\hline
\end{tabular}

Fonte: Coleta de dados

$(\mathrm{a}, \mathrm{b}, \mathrm{c})$ diferenças entre grupos

$* \mathrm{p}<0,05$

Com relação às "funções básicas", o cluster 3 se diferenciou dos demais, apresentando a menor média $(\bar{x}=3,70)$, ou seja, é o grupo que menos se interessa por essas funções. Por outro lado, o fator "funções avançadas" apresentou médias significativas para os três grupos. O cluster 1 se diferencia dos demais e possui a maior média $(\bar{x}=3,56)$, indicando que esse grupo apresenta um interesse maior que os demais pelas "funções avançadas". O grupo 2 também se diferencia dos demais, apresentando a menor média $(\bar{x}=2,41)$, sendo o grupo que menos se interessa pelas "funções avançadas".

Os três grupos também se diferenciam quanto ao fator "tipo de plano", sendo que o Cluster 1 apresentara a média mais alta $(\bar{x}=4,49)$ em relação aos demais, indicando que esse grupo é o que mais se interessa em saber sobre os planos e preços de aparelhos. Por outro lado, o grupo 3 é o que menos se interessa por esse fator em relação aos demais, apresentando a menor média $(\bar{x}=3,92)$.

Para o fator "operadora", o cluster 2 é o único que se diferencia em relação aos demais grupos, apresentando a maior média $(\bar{x}=3,93)$, indicando que esse grupo é o que mais valoriza os serviços prestados pela operadora. O cluster 1 é o que mais dá importância ao fator “design", com média $(\bar{x}=3,93)$, diferenciando-se nesse fator em relação aos demais grupos. 
A maior parte dos benefícios buscados não apresentou poder de discriminação entre os grupos. Esses benefícios incluíram os relacionados às "funções básicas", "internet", "visor colorido", "tipo de tecnologia", "tipo de plano" e "operadora". Os benefícios que apresentaram algum poder de discriminação entre os grupos foram "design", "câmera fotográfica", "mp3 player", "área de cobertura" e "marca do aparelho".

O teste Qui quadrado $\left(\chi^{2}\right)$ para o atributo "design" e o benefício "ser discreto" sugere maior concentração no grupo 2 , sendo que o grupo 1 foi o que menos associou o "design" a esse benefício. O grupo 1 se diferencia principalmente dos outros no maior percentual para a associação entre os atributos "câmera fotográfica" e "mp3 player" ao beneficio ser "moderno". A "área de cobertura", associada ao beneficio "permitir que me encontrem" diferencia o grupo 2 dos demais grupos. A preocupação de que o aparelho comprado seja "moderno" aparece como uma característica que diferencia o grupo dos 3 dos demais grupos.

A Tabela 03 apresenta de forma resumida os valores associados ao Qui Quadrado $\left(\chi^{2}\right)$ para essas variáveis e seu nível de significância.

Tabela 03 - Teste Qui-Quadrado para Benefícios Buscados e Grupos de Consumidores

\begin{tabular}{l|l|c|c|c|c|c}
\hline \multirow{2}{*}{ Atributo } & \multirow{2}{*}{ Benefício } & \multicolumn{2}{|c|}{ GRUPOS ENCONTRADOS } & \multirow{2}{*}{$\chi^{2}$} & \multirow{2}{*}{ p } \\
\cline { 3 - 5 } & & Cluster 1 & Cluster 2 & Cluster 3 & & \\
\hline Design & Ser discreto & $26,6 \%$ & $42,7 \%$ & $30,8 \%$ & 8,704 & $0,013^{*}$ \\
\hline Câmera & Ser moderno & $40 \%$ & $31,1 \%$ & $28,9 \%$ & 8,441 & $0,015^{*}$ \\
\hline Mp3 & Ser moderno & $44,8 \%$ & $29,9 \%$ & $25,4 \%$ & 7,932 & $0,019^{*}$ \\
\hline Cobertura & $\begin{array}{l}\text { Permitir que me } \\
\text { encontrem }\end{array}$ & $30,1 \%$ & $50,3 \%$ & $19,7 \%$ & 3,729 & $0,035^{*}$ \\
\hline Aparelho & Ser moderno & $30,4 \%$ & $30,4 \%$ & $39,3 \%$ & 10,105 & $0,006^{*}$ \\
\hline \\
Fonte: Coleta de dados \\
$*$ p $<0,05$
\end{tabular}

Com base nas relações estabelecidas entre as variáveis do modelo proposto e os clusters encontrados, foi delineado o perfil dos grupos da taxonomia, descritos a seguir.

\subsubsection{Interessados no Equipamento - Tecnológicos}

O cluster dos Tecnológicos (grupo 1) é composto por 102 consumidores e foi assim denominado por apresentar média mais alta na preferência por funções avançadas $(\bar{x}=3,56)$, diferenciando-se estatisticamente dos demais. Também apresenta a média mais alta em relação à importância dada aos atributos do fator "tipo de plano" $(\bar{x}=4,49)$. Os Tecnológicos apresentam uma preferência maior por atributos relacionados ao "design" $(\bar{x}=4,58)$, diferindo estatisticamente dos demais. A importância dada para o fator "funções básicas" é relativamente alta $(\bar{x}=4,60)$ e difere estatisticamente apenas do grupo 3 (Utilitários).

É importante destacar também que esse grupo se diferencia dos outros em relação à posse e utilização de funções avançadas, apresentando as médias mais altas $(\bar{x}=7,64$ e $\bar{x}=$ 2,52 , respectivamente). Além disso, os Tecnológicos apresentam uma média estatisticamente mais alta de posse de funções intermediárias $(\bar{x}=3,47)$, em comparação ao grupo dos Focados no Serviço $(\bar{x}=2,92)$, sem se diferenciar do grupo dos Utilitários $(\bar{x}=3,33)$. Quanto à utilização de funções básicas, o grupo 1 apresenta uma média estatisticamente superior $(\bar{x}=$ 4,11), também se diferenciando do grupo $3(\bar{x}=3,81)$.

Esse grupo também sustenta o maior percentual na relação entre a preferência pelos atributos "câmera fotográfica" $\left(\chi^{2}=8,441, p=0,015\right)$ e "mp3 player" $\left(\chi^{2}=7,932, p=0,019\right)$ e o beneficio "ser moderno".

\subsubsection{Interessados no Serviço Prestado pela Operadora - Focados no Serviço}


O cluster dos Focados no Serviço (grupo 2) é composto por 138 consumidores, 43,9\% da amostra, que se caracteriza pela importância dada ao fator "operadora" $(\bar{x}=3,93)$, diferenciando-se dos outros dois grupos. Entretanto, é o grupo com o nível mais baixo de importância dada ao fator "funções avançadas" $(\bar{x}=2,41)$, diferenciando-se dos demais. A relevância dada aos atributos do fator "tipo de plano" é moderada em relação aos outros grupos $(\bar{x}=4,24)$.

Em comparação ao primeiro grupo, os Focados no Serviço diferenciam-se na posse de funções intermediárias, com uma média mais baixa $(\bar{x}=2,92)$, em relação ao grupo $1(\bar{x}=$ $3,47)$.

O atributo "área de cobertura" relacionado ao benefício "permitir que me encontrem", apresentou um percentual estatisticamente superior em relação aos outros dois grupos $\left(\chi^{2}=\right.$ $3,729, \mathrm{p}=0,035)$. Além disso, o atributo "design", relacionado ao benefício "ser discreto" também apresentou um percentual estatístico superior em relação aos demais $\left(\chi^{2}=8,704\right.$, $\mathrm{p}=0,013)$.

\subsubsection{Utilitários}

O cluster dos Utilitários (grupo 3) é composto por 74 consumidores, 23,6\% da amostra e apresenta o nível mais baixo de preferência pelas "funções básicas" $(\bar{x}=3,70)$, diferenciando-se dos demais. A importância dada às "funções avançadas" é estatisticamente superior ao grupo dos Focados no Serviço e inferior ao grupo dos Tecnológicos, indicando um nível de interesse mediano por esses atributos em comparação aos outros grupos.

Mesmo com médias relativamente altas de importância para o fator "tipo de plano", os Utilitários dão menos importância para esses atributos em comparação com os outros grupos $(\bar{x}=3,92)$.

Quanto ao fator "operadora", os Utilitários se diferenciam dos Focados no Serviço apresentando uma média estatisticamente menor $(\bar{x}=3,63)$, mas não se diferenciam dos Tecnológicos, indicando que os grupos 1 (Tecnológicos) e 3 (Utilitários) valorizam menos esses atributos em comparação com o grupo 2 (Focados no Serviço).

Com relação ao fator "design", o grupo apresenta uma média estatisticamente menor do que o grupo $1(\bar{x}=3,83)$, mas não chega a se diferenciar do grupo 2 .

Os Utilitários têm a mais baixa concentração na importância dada às "funções avançadas" (mp3 e câmera) relacionadas ao beneficio "ser moderno", e na importância dada à "cobertura" relacionada ao beneficio "permitir que me encontrem". Entretanto, valorizam a modernidade do aparelho $\left(\chi^{2}=10,105, p=0,006\right)$.

\section{CONCLUSÕES}

Com os resultados da pesquisa foi possível caracterizar os grupos encontrados entre os consumidores pesquisados com uma útil taxonomia tendo como base a preferência por atributos, sobretudo, para a faixa etária de jovens estudantes que parece representar uma parte importante do universo de usuários de telefonia celular. De qualquer forma, não é demais salientar que não existe a pretensão de generalização dos achados empíricos reportados aqui. Como já foi mencionado, a pesquisa tem caráter fundamentalmente exploratório (MALHOTRA, 2001). Entretanto, algumas relações podem ser inferidas com base nos perfis dos grupos apresentados no trabalho.

Os atributos considerados mais relevantes no processo de escolha foram identificados na fase qualitativa, juntamente com seus benefícios associados. Nesta fase, observou-se que os principais critérios de escolha dos usuários não foram atributos técnicos, mas sim de ordem estética e relacionados ao serviço da operadora como "design", "tamanho do aparelho", "preço", "marca do aparelho" ou "ter visor colorido". Os atributos básicos também foram citados como, por exemplo, "agenda telefônica", "despertador", "mensagem de texto" e 
"vibracall". As únicas funções avançadas citadas foram "câmera fotográfica", "acesso à internet" e "mp3 player". Além disso, alguns serviços relacionados à operadora foram considerados importantes como o preço, a área de cobertura, tipo de tecnologia, tipo de plano e operadora.

Os benefícios buscados nesses atributos foram divididos em dois grupos: concretos e simbólicos. Os benefícios simbólicos relacionam-se à aparência, status, estar na moda e fazer parte do grupo em que convive. Já os benefícios concretos são relacionados à praticidade, à facilidade de uso, à economia financeira e à qualidade do produto ou serviço. Na fase quantitativa, foi elaborada uma matriz para identificar as ligações entre atributos e benefícios.

Foram encontrados três grupos de jovens consumidores de telefonia celular com base na preferência por atributos. O primeiro grupo foi denominado de Tecnológicos, pois se diferenciam dos outros grupos principalmente pelo interesse que têm nas funções avançadas e no design. Os consumidores deste grupo relacionam os atributos "câmera fotográfica" e "mp3 player" (funções avançadas) ao beneficio "ser moderno", o que também tende a explicar em parte, o interesse desses consumidores por essas funções.

Os indivíduos do segundo grupo (Focados no Serviço) se caracterizam pela valorização ao serviço prestado pela operadora, sendo o grupo com nível mais baixo de importância dada ao fator "funções avançadas", mas nem por isso deixam de se interessar pelo design do equipamento, pois o atributo "design", relacionado ao benefício "ser discreto" apresentou um percentual estatístico superior em relação aos demais, juntamente com a importância dada à área de cobertura, relacionado ao benefício "ser encontrado".

Os jovens do grupo 3 (Utilitários) apresentam o nível mais baixo de preferência pelas funções básicas e um interesse mediano pelas funções avançadas. Os Utilitários têm a mais baixa concentração na importância dada às funções avançadas ("mp3 player" e "câmera") relacionadas ao beneficio "ser moderno", e na importância dada à "cobertura" relacionada ao beneficio "permitir que me encontrem". Entretanto, valorizam a modernidade do aparelho, mesmo que este não tenha funções consideradas avançadas.

De maneira geral, o estudo expressa a riqueza de informações provenientes das interações entre as bases comportamentais de segmentação na descrição de mercados assumidos como altamente homogêneos, como parece ser o caso do grupo de estudantes que foi objeto de análise.

\section{Referências}

AAKER, D. A.; KUMAR, V.; DAY, G. S. Pesquisa de Marketing. São Paulo: Atlas, 2004.

ALPERT, M.I. Identification of determinant attributes: a comparison of methods. Journal of Marketing Research, Vol. 8, p. 184-191, maio, 1971.

BAGOZZI, R.P.; DHOLAKIA, U. Goal setting and goal striving in consumer behavior. Journal of Marketing. v.63, p.19-32, 1999.

BETTMAN, J. R.; LUCE, M. F.; PAYNE, J. W. Constructive consumer choice processes. Journal of Consumer Research, v.25, p.187-214, dez.. 1998.

CHERNEV, A. Context effects without a context: attribute balance as a reason for choice. Journal of Consumer Research, v.32, n.2, p.213-224, set. 2005.

CHURCHILL, JR, Gilbert A. Marketing Research: Methodological Foundations, $7^{\text {th }}$ Ed. Fort Worth: Dryden, 1999.

COUPEY, E. Restructuring: constructive processing of information displays in consumer choice. Journal of Consumer Research, v. 21, p. 83-99, jun. 1994. 
DESAY, K. K.; HOYER, W. D. Descriptive characteristics of memory-based consideration sets: influence of usage occasion frequency and usage location familiarity. Journal of Consumer Research. V. 27, p. 309-322, 2000.

DESAY, K. K.; RATNESHWAR, S. Consumer perceptions of product variants positioned on atypical attributes. Academy of Marketing Science Journal. v. 31, n. 1, p.22-35, 2003

DHAR, R.; NOWLIS, S. M.; SHERMAN, S. J. Comparison effects on preference construction. Journal of Consumer Research. v.26, n.3, p.293-306, dez. 1999.

ENGEL, J. F; BLACKWELL, R D; MINIARD, P. W. Comportamento do Consumidor. 8 ed. Rio de Janeiro: LTC, 2000.

FERREIRA, A. C. A. dos S. O comportamento do consumidor jovem. VI Semead, São Paulo, 2003. Anais.

GUTMAN, J. Means-end chains as goals hierarchies. Psychology \& Marketing. v.14, n.6, p.545-559, 1997.

HAIR, J.; ANDERSON, R.; TATAHAN, R.; BLACK, W. Multivariate Data Analysis, Prentice Hall, 2005.

HSM Management. A marca na palma da mão. São Paulo: Abril, mar-abr.2006.

HUFFMAN, C.; HOUSTON, M. J. Goal-oriented experiences and the development of knowledge. Journal of Consumer Research, v.20, n.2, p. 190-208,1993.

LEFKOFF-HAGIIJS, R.; MANSON, C. H. Characteristic, beneficial, and image attributes in consumer judments of similarity and preference. Journal of Consumer Research, v.20, n.1, p. 100-110, jun. 1993.

LIN, C.; FU, H. Exploring logic construction on MECs to enhance marketing strategy. Marketing Intelligence \& Planning. V.19, p. 362-367, 2001.

MACKENZIE, S. The role of attention in mediating the effect of advertising on attribute importance. Journal of Consumer Research, v. 13, p.174-195, set. 1986.

MALHOTRA, N.K. Marketing research: an applied orientation. Upper Saddle River: Prentice-Hall, 2001.

MOWEN, J. C.; MINOR, M. S. Comportamento do consumidor. São Paulo: Prentice-Hall, 2003.

PÁDUA JÚNIOR, F. P.; PRADO, P. H. A Adoção de Inovações em Produtos de Alta Tecnologia Por Jovens: O Caso do Telefone Celular. XXIX ENANPAD, Brasília, 2005. Anais.

RATNESHWAR, S.; HUFFMAN, C.; MICK, D. G. The why of consumption: contemporary perspectives on consumer motives, goals and desires. London: Routledge, 2000 .

SHETH, J. N.; MITTAL, B.; NEWMAN, B. I. Customer behavior - consumer behavior and beyond. Orlando: The Dryden Press, 2001.

SOLOMON, M. R. O comportamento do consumidor: comprando, possuindo e sendo. 5. ed. Porto Alegre: Bookman, 2002.

TELEBRASIL. Site da TELEBRASIL. Disponível em: <http://www.telebrasil.gov.br> Acesso em 09 jul. 2006.

TELECO. Site da Teleco. Disponível em: <http://www.teleco.com.br/> Acesso em 14 jun. 2006.

THOMPSON, D. V.; HAMILTON, R. W.; RUST, R. T. Feature fatigue: when product capabilities become too much of a good thing. Marketing Science Institute, 2005.p.27-47. 
VEJA. Jovens. São Paulo: Abril, v. 37, n. 32, Ed. 1904, maio 2005.

VRIENS, M.; TER HOFSTEDE, F. Linking attributes, benefits and consumer values: a powerful approach to market segmentation, brand positioning and advertising strategy. Marketing Research, v.12, p.5-10, 2000.

WEINSTEIN, A. Segmentação de Mercado. São Paulo: Atlas, 1995.

ZAICHKOWSKY, J. L. Measuring the involvement construct. Journal of Consumer Research, v. 12, p. 341-352, dez. 1985.

ZEITHAML, V. A. Consumer perceptions of price, quality, and value: a means-end model and synthesis of evidence. Journal of Marketing, v.52, n.2, p.2-22, July 1988. 